\title{
Three-body charmless hadronic $B$ decays at BABAR
}

\section{Thomas LATHAM* (On behalf of the BABAR Collaboration)}

University of Warwick

E-mail: T.Lathamewarwick.ac.uk

We present recent results from the $B A B A R$ experiment on three-body charmless hadronic $B$ decays. The results include searches for the decay of charged $B$ mesons to the final state $K_{S}^{0} K_{S}^{0} \pi^{+}$and for neutral $B$ mesons to $K_{S}^{0} K_{S}^{0} \pi^{0}, K_{S}^{0} K_{S}^{0} \eta$ and $K_{S}^{0} K_{S}^{0} \eta^{\prime}$. We also present a Dalitz-plot analysis of charged $B$ decays to the final state $\pi^{+} \pi^{+} \pi^{-}$. All analyses use a sample of approximately 465 million $B \bar{B}$ pairs collected by the BABAR detector at the PEP-II asymmetric energy $B$ Factory at SLAC.

European Physical Society Europhysics Conference on High Energy Physics July 16-22, 2009

Krakow, Poland

${ }^{*}$ Speaker. 


\section{Introduction}

The decays of $B$ mesons to charmless three-body final states can include contributions from both tree level and loop (or penguin) processes. The interference between these different contributions can give rise to direct $C P$ violation. The interference between different intermediate states in the Dalitz plot means that relative phases as well as magnitudes are observable. Utilising the Dalitz-plot analysis techniques and in some cases combining these with time-dependent analysis permits measurements of all three angles of the CKM Unitarity Triangle. Some of the possible intermediate resonances are not particularly well understood, particularly the scalars. By studying as many three-body decays as possible and determining where certain resonances contribute it provides more information on their nature.

\section{Analysis Techniques}

The results presented here are all based on a data sample of 465 million $B \bar{B}$ pairs collected by the BABAR detector [1] at the PEP-II asymmetric energy $B$ Factory at the SLAC National Accelerator Laboratory. The $B$ meson candidates are characterised using topological and kinematic variables. The former take advantage of the fact that the centre of mass energy $\sqrt{s}$ is just above the threshold for $B \bar{B}$ production and therefore the $B$ mesons are produced almost at rest in that frame. The topological variables are generally combined in a multivariate analyser (MVA), such as a neural network, in order to maximise their discriminating power. The kinematic variables are $m_{\mathrm{ES}}=\sqrt{\frac{s}{4}-\vec{p}_{B}^{\star 2}}$ and $\Delta E=E_{B}^{\star}-\frac{\sqrt{s}}{2}$, where $\left(E_{B}^{\star}, \vec{p}_{B}^{\star}\right)$ is the $B$ four-momentum in the centre of mass frame. The topological MVA, $m_{\mathrm{ES}}$ and $\Delta E$ are used as inputs to a maximum likelihood fit.

\section{Search for $B^{+} \rightarrow K_{S}^{0} K_{S}^{0} \pi^{+}$}

The decay $B^{+} \rightarrow K_{S}^{0} K_{S}^{0} \pi^{+}$proceeds mainly via the $b \rightarrow d$ penguin transition, which is suppressed in the Standard Model (SM). However, BABAR has recently observed the isospin related mode $B^{+} \rightarrow K^{+} K^{-} \pi^{+}$with a branching fraction of $5 \times 10^{-6}[$ [2]. This analysis also reported an unexpected enhancement in the $K^{+} K^{-}$invariant mass spectrum around $1.5 \mathrm{GeV}$. Similar structures have also been seen in analyses of $B \rightarrow K K K$ and $B \rightarrow K \pi \pi$ decays and have been dubbed the $f_{X}(1500)$, see for example [3]. If the $f_{X}(1500)$ has even spin and its decays follow isospin symmetry one would expect the rate to $K_{S}^{0} K_{S}^{0}$ to be $50 \%$ that to $K^{+} K^{-}$. Consequently any observation or lack of a similar structure in $B^{+} \rightarrow K_{S}^{0} K_{S}^{0} \pi^{+}$could provide information on the spin and/or quark content of this state. The BABAR analysis, described in detail in [ $\llbracket$, makes no prior assumption on the Dalitz-plot distribution and therefore includes all possible resonant and nonresonant decay modes. The analysis finds no evidence of signal. The branching fraction and upper limit are given in Table 1. The lack of signal disfavours models where the $f_{X}(1500)$ has even spin and decays with isospin symmetry. More data are required to clarify this situation.

\section{Search for $B^{0} \rightarrow K_{S}^{0} K_{S}^{0} \pi^{0}, K_{S}^{0} K_{S}^{0} \eta, K_{S}^{0} K_{S}^{0} \eta^{\prime}$}

The search for these decay modes is also motivated by the mystery of the $f_{X}(1500)$ as outlined in Section 3. There is an additional motivation for studying these modes, which is that they provide 


\begin{tabular}{lcc}
\hline Decay Mode & Branching Fraction $\left(10^{-7}\right)$ & $90 \%$ CL UL $\left(10^{-7}\right)$ \\
\hline$B^{+} \rightarrow K_{S}^{0} K_{S}^{0} \pi^{+}$ & $2.5 \pm 2.4 \pm 0.9$ & 5 \\
$B^{0} \rightarrow K_{S}^{0} K_{S}^{0} \pi^{0}$ & $2.7_{-3.7}^{+4.2} \pm 0.6$ & 9 \\
$B^{0} \rightarrow K_{S}^{0} K_{S}^{0} \eta$ & $2.1_{-3.8}^{+4.7} \pm 1.2$ & 10 \\
$B^{0} \rightarrow K_{S}^{0} K_{S}^{0} \eta^{\prime}$ & $5.7_{-6.5}^{+8.0} \pm 3.4$ & 20 \\
\hline
\end{tabular}

Table 1: Branching fraction central values and 90\% confidence level upper limits for the decays $B^{+} \rightarrow$ $K_{S}^{0} K_{S}^{0} \pi^{+}, B^{0} \rightarrow K_{S}^{0} K_{S}^{0} \pi^{0}, K_{S}^{0} K_{S}^{0} \eta$ and $K_{S}^{0} K_{S}^{0} \eta^{\prime}$. The first (second) uncertainty is statistical (systematic).

the possibility of time-dependent $C P$ asymmetry measurements. Each of the final states is itself a $C P$ eigenstate since it consists of three $C P$ eigenstate particles, two of which are identical [5]. Consequently there is no need for an isospin or Dalitz-plot analysis in order to separate the different contributions. In this analysis, described in detail in [6], $\eta$ candidates are reconstructed in their decays to $\gamma \gamma$ and three pions and $\eta^{\prime}$ candidates are reconstructed decaying to $\eta \pi \pi$ and $\rho \gamma$. No significant signals are seen. Table 1 contains the central values of the branching fractions for each of the modes along with the Bayesian 90\% confidence level upper limits that have been calculated. The small signal yield in these modes unfortunately precludes performing a time-dependent analysis with the present statistics.

\section{Dalitz-plot Analysis of $B^{+} \rightarrow \pi^{+} \pi^{+} \pi^{-}$}

Studies of the Dalitz-plot structure of $B^{+} \rightarrow \pi^{+} \pi^{+} \pi^{-}$decays are importand for several reasons. It is in principle possible to extract the CKM angle $\gamma$ from the interference of $B^{+} \rightarrow \chi_{c 0} \pi^{+}$and other modes in the $B^{+} \rightarrow \pi^{+} \pi^{+} \pi^{-}$Dalitz plot, such as $B^{+} \rightarrow \rho^{0} \pi^{+}$. The information on the signal Dalitz-plot model in this mode provides important input to the analysis of $B^{0} \rightarrow \pi^{+} \pi^{-} \pi^{0}$, which is used to measure the CKM angle $\alpha$. As with all charmless threebody modes the measurements of branching fractions and $C P$ asymmetries help to test QCD factorisation and other effective theories. In this analysis, described in detail in [7], the signal Dalitz-plot isobar model contains contributions from $\rho^{0}(770), \rho^{0}(1450), f_{2}(1270), f_{0}(1370)$ and a nonresonant component. The fit projections on the Dalitz plot co-ordinates are shown in Figure 11 and the fit results are shown in Table 2 . The Dalitz plot is mainly dominated by the $\rho^{0}(770)$. The $\chi_{c 0}$ and $\chi_{c 2}$ components are found not to be significant, precluding measurement of $\gamma$ with the present statistics.

\section{Acknowledgments}

I would like to thank the principal authors of the analyses presented here and Tim Gershon for their guidance in the preparation of this document and the conference presentation. This work is supported in part by the United Kingdom's Science and Technology Facilities Council and the US Department of Energy under contract number DE-AC02-76SF00515.

\section{References}

[1] B. Aubert et al. [BABAR Collaboration], The BABAR Detector, Nucl. Instrum. Meth. A 479 (2002) 1 [arXiv:hep-ex/0105044]. 

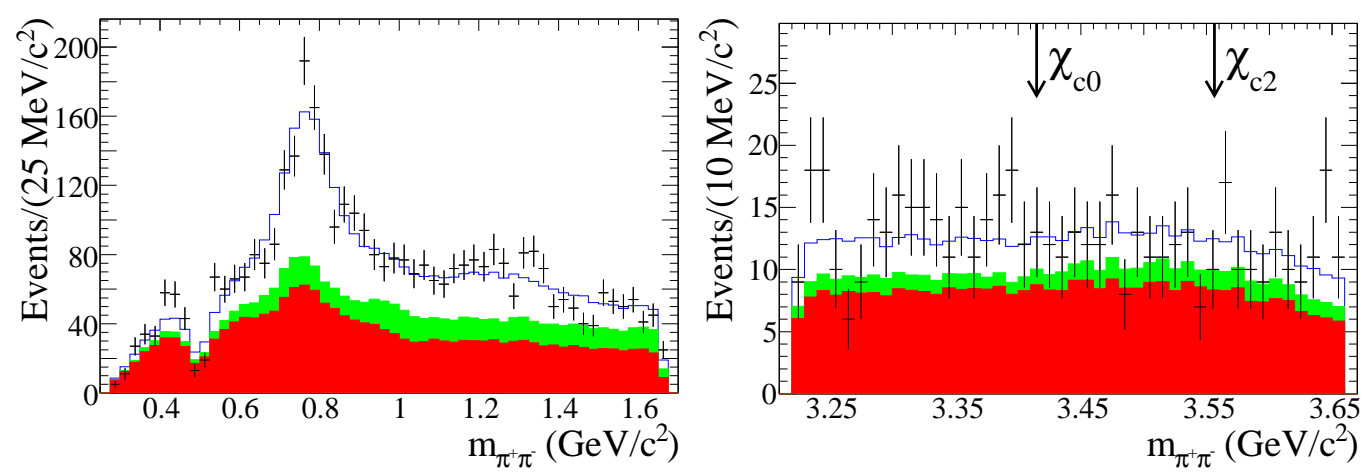

Figure 1: The fit projections onto the $\pi^{+} \pi^{-}$invariant mass in the low mass region (left) and the region where the $\chi_{c 0}$ and $\chi_{c 2}$ are expected (right).

\begin{tabular}{lcc}
\hline \hline Mode & $\mathscr{B}\left(B^{+} \rightarrow\right.$ Mode $)\left(10^{-6}\right)$ & $A_{C P}(\%)$ \\
\hline$\pi^{+} \pi^{-} \pi^{-}$Total & $15.2 \pm 0.6 \pm 1.2_{-0.3}^{+0.4}$ & $+3.2 \pm 4.4 \pm 3.1_{-2.0}^{+2.5}$ \\
\hline$\rho^{0}(770) \pi^{+}, \rho^{0}(770) \rightarrow \pi^{+} \pi^{-}$ & $8.1 \pm 0.7 \pm 1.2_{-1.1}^{+0.4}$ & $+18 \pm 7 \pm 5_{-14}^{+2}$ \\
$\rho^{0}(1450) \pi^{+}, \rho^{0}(1450) \rightarrow \pi^{+} \pi^{-}$ & $1.4 \pm 0.4 \pm 0.4_{-0.7}^{+0.3}$ & $-6 \pm 28 \pm 20_{-35}^{+12}$ \\
$f_{2}(1270) \pi^{+}, f_{2}(1270) \rightarrow \pi^{+} \pi^{-}$ & $0.9 \pm 0.2 \pm 0.1_{-0.3}^{+0.3}$ & $+41 \pm 25 \pm 13_{-8}^{+12}$ \\
$f_{0}(1370) \pi^{+}, f_{0}(1370) \rightarrow \pi^{+} \pi^{-}$ & $2.9 \pm 0.5 \pm 0.5_{-0.5}^{+0.7}(<4.0)$ & $+72 \pm 15_{1} \pm 14_{-8}^{+7}$ \\
$\pi^{+} \pi^{-} \pi^{-}$nonresonant & $5.3 \pm 0.7 \pm 0.6_{-0.5}^{+1.1}$ & $-14 \pm 14 \pm 7_{-3}^{+17}$ \\
$f_{0}(980) \pi^{+}, f_{0}(980) \rightarrow \pi^{+} \pi^{-}$ & $<1.5$ & - \\
$\chi_{c 0} \pi^{+}, \chi_{c 0} \rightarrow \pi^{+} \pi^{-}$ & $<0.1$ & - \\
$\chi_{c 2} \pi^{+}, \chi_{c 2} \rightarrow \pi^{+} \pi^{-}$ & $<0.1$ & - \\
\hline \hline
\end{tabular}

Table 2: Branching fraction and $C P$ asymmetries for the various contributions to the $\pi^{+} \pi^{-} \pi^{-}$Dalitz plot. Where a component is not signficant a Bayesian $90 \%$ confidence level upper limits is given. The first (second) uncertainty is statistical (systematic).

[2] B. Aubert et al. [BABAR Collaboration], Observation of the Decay $B^{+} \rightarrow K^{+} K^{-} \pi^{+}$Phys. Rev. Lett. 99 (2007) 221801 [arXiv:0708.0376 [hep-ex]].

[3] A. Garmash et al. [Belle Collaboration], Dalitz analysis of the three-body charmless decays $B^{+} \rightarrow K^{+} \pi^{+} \pi^{-}$and $B^{+} \rightarrow K^{+} K^{+} K^{-}$Phys. Rev. D 71 (2005) 092003 [arXiv:hep-ex/0412066].

[4] B. Aubert et al. [BABAR Collaboration], Search for the decay $B^{+} \rightarrow K_{S}^{0} K_{S}^{0} \pi^{+}$Phys. Rev. D 79 (2009) 051101 [arXiv:0811.1979 [hep-ex]].

[5] T. Gershon and M. Hazumi, Time-dependent $C P$ violation in $B^{0} \rightarrow P^{0} P^{0} X^{0}$ decays Phys. Lett. B 596 (2004) 163 [arXiv: hep-ph/0402097].

[6] B. Aubert et al. [BABAR Collaboration], Search for $B^{0}$ Meson Decays to $\pi^{0} K_{S}^{0} K_{S}^{0}, \eta K_{S}^{0} K_{S}^{0}$, and $\eta^{\prime} K_{S}^{0} K_{S}^{0}$ Phys. Rev. D 80 (2009) 011101 [arXiv:0905.0868 [hep-ex]].

[7] B. Aubert et al. [BABAR Collaboration], Dalitz Plot Analysis of $B^{ \pm} \rightarrow \pi^{ \pm} \pi^{ \pm} \pi^{\mp}$ Decays Phys. Rev. D 79 (2009) 072006 [arXiv:0902.2051 [hep-ex] ]. 